\title{
Enhancement of Fetal Lung Surfactant Production by Aminophylline
}

\author{
ALEX SEVANIAN, CARL GILDEN, SOLOMON A. KAPLAN, AND CYNTHIA T. BARRETT \\ University of California at Los Angeles, Center for the Health Sciences, Los Angeles, California, USA
}

\begin{abstract}
Summary
Antepartum administration of aminophylline to pregnant rabbits resulted in accelerated formation of phospholipids regarded to be important components of pulmonary surfactant. Increases in the tissue content and synthesis of saturated phosphatidylcholine and phosphatidylinositol were found in lung slice preparations obtained from fetuses previously treated with aminophylline. Synthesis of phospholipids was measured using labeled palmitate, oleate, and glucose as precursors. The results showed increased de novo production of these lipids, and that glucose and glycogen may serve as important precursors. There was a significant reduction in triglyceride content while free fatty acids increased suggesting increased lipolysis in the aminophylline-treated groups, but this did not measurably affect the rate of labeled palmitate incorporation into total phospholipids.
\end{abstract}

\section{Speculation}

Prenatal treatment with aminophylline may prove to be an effective means of preventing idiopathic respiratory distress syndrome. This method could be an effective and safe means of accelerating pulmonary surfactant production before premature delivery.

Maturation of fetal mammalian lungs can be accelerated by antenatal administration of glucocorticoids. Decreased morbidity of prematurely born infants has been reported in human infants (22) and experimental evidence of increased pulmonary surfactant production after glucocorticoid administration is well documented $(10,11,20)$. The effects of cortisol on fetal lung phospholipid metabolism have been shown to involve increased de novo synthesis of phosphatidylcholine (11) utilizing, in part, lung glycogen as a substrate (12). Furthermore, a good correlation exists between elevated levels of cyclic $3^{\prime}, 5^{\prime}$-adenosine monophosphate (cAMP) and an acceleration of phospholipid formation in fetal lungs of animals previously treated with cortisol (3).

Notwithstanding the beneficial effects, glucocorticoid therapy for lung immaturity has not been uniformly accepted because of possible deleterious effects when pharmacologic doses are used ( 7 , $18,27)$ including a recent report of increased incidence of maternal infection after glucocorticoid administration (30). For these reasons, a search for other means for pharmacologic intervention is appropriate. In recent studies, it has been demonstrated that accelerated maturation of fetal lungs may occur after administration of aminophylline in a manner comparable to that observed for glucocorticoids $(3,19)$. There also occurs an increase in lung tissue cAMP concentration, which correlates with increased phospholipid synthesis (3). Improved survival of prematurely delivered rabbits after aminophylline administration was recently reported (2). This increased survival was associated with pulmonary physiologic and biochemical indices which were significantly more mature than in control or cortisol treated litters.

Based on these findings, the authors have conducted a series of studies analyzing the biochemical effects of aminophylline on the production of pulmonary surfactant phospholipids. These studies are a part of a continuing effort to evaluate the metabolic effects, efficacy, and potential toxicity of aminophylline as a preventive agent for idiopathic respiratory distress syndrome.

\section{MATERIALS AND METHODS}

The fetal rabbit lung slice model was used. Details of gestational timing as well as treatment schedule are described elsewhere (3). On the 27 th day of gestation, the fetuses were delivered by cesarean section with the doe under general anesthesia. The fetal lungs were excised and placed in cold Krebs-Ringer buffer as described by O'Neil and Tierney (23). The lungs were rinsed in the same buffer, blotted dry, and slices of 1000 micron thickness prepared. Approximately $300 \mathrm{mg}$ tissue were transferred into metabolic flasks containing $4.5 \mathrm{ml} \mathrm{Krebs-Ringer} \mathrm{bicarbonate}$ buffer and $5 \%$ bovine serum albumin (Pentex fraction V). Incorporation of radiolabeled substrates into various tissue lipids was measured utilizing the following compounds in the concentrations specified: $32 \mu \mathrm{M}^{14} \mathrm{C}$-L-palmitic acid $(0.65 \mu \mathrm{Ci} / \mu$ mole $), 32 \mu \mathrm{M}^{3} \mathrm{H}-9$, 10 oleic acid $(7.8 \mu \mathrm{Ci} / \mu$ mole $)$, and $35 \mathrm{mM}^{14} \mathrm{C} \cdot \mathrm{U}$ glucose $(18.5$ $\mu \mathrm{Ci} / \mu \mathrm{mole})$. Radiochemicals were purchased from New England Nuclear Corporation. Fatty acid substrates were bound to albumin. as their sodium salts by titration with $0.1 \mathrm{~N} \mathrm{NaOH}$ and heating to $80^{\circ} \mathrm{C}$. After cooling, the lipids were added to a $5 \%$ solution of albumin in Krebs-Ringer bicarbonate buffer. The flasks were weighed before and after addition of tissue and tissue weight was determined by the weight difference of flasks. After equilibration for $45 \mathrm{sec}$ with $95 \% \mathrm{O}_{2}$ and $5 \% \mathrm{CO}_{2}$, the flasks were incubated for $90 \mathrm{~min}$ in a Dubnoff metabolic shaker bath set at $37^{\circ} \mathrm{C}$ at 120 oscillations/min. After incubation, the tissue was transferred to buffer at $4^{\circ} \mathrm{C}$, washed several times, lyophilized at $-30^{\circ} \mathrm{C}$ and stored at $-20^{\circ} \mathrm{C}$ under nitrogen until analyzed. In some flasks in each group, $0.5 \mathrm{ml}$ quarternary amine was added to the center well and $2 \mathrm{ml}$ of $6 \%$ perchloric acid to the outer compartment (containing the buffer and tissue) by means of a needle, while the flasks remained sealed. These flasks were reincubated for $60 \mathrm{~min}$ to collect $\mathrm{CO}_{2}$ in the center well. The quarternary amine was transferred to vials containing ethanol and Aquasol (New England Nuclear Corp.). Radioactivity was measured in a liquid scintillation counter (Nuclear Chicago Isocap 300).

Tissue glycogen content was determined before and after incubation by the method of Van Handel (31). Glucose content in the medium was measured before and after incubation by the method of Washko and Rice (32). Lactate content in the medium was measured by the method of Hohorst (16), and tissue lactate by the method of Davidson and Berliner (8).

Lipid analysis involved extraction of total lipids from lyophilized tissue by a modified Bligh and Dyer procedure (6) using a Polytron homogenizer (Brinkman Instruments). Phospholipids were separated from neutral lipids by silicic acid column chromatography. The phospholipids were fractionated by two-dimensional thin-layer chromatography on silica gel-H coated plates (Analtech Labs) using chloroform-methanol-ammonia (65:35:5) 
in the first dimension and chloroform-acetone-methanol-acetic acid-water (5:3:2:0.5:1) in the second dimension. Regions corresponding to phosphatidylcholine, sphingomyelin, phosphatidylglycerol, and phosphatidylinositol were recovered and quantitated on the basis of phosphorus content by the method of Bartlett (4) and by radioactivity. Saturated phosphatidylcholine was prepared from an aliquot of isolated phosphatidylcholine by the method of Shimojo et al. (29) and measurements made as described above. The neutral lipids were fractionated by thin-layer chromatography using petroleum ether-ethyl ether-acetic acid (80:20:1). Regions corresponding to triglycerides and free fatty acids were collected, radioactivity determined as described previously, and content estimated after conversion to fatty acid methyl esters. This was accomplished by using $0.5 \mathrm{~N} \mathrm{HCl}$-methanol or ethereal diazomethane, and the esters were quantitated in a gas-liquid chromatograph (Perkin-Elmer 881 ) fitted with a $12 \%$ diethyieneglycol succinate column (Supelco Corp). Fatty acid methyl ester standards were obtained from Applied Sciences Labs.

Values for all chemical determinations were expressed per unit wet weight. The ratio of wet to dry weight did not vary significantiy between the control and study groups. Molar incorporation of labeled precursors into various products was calculated on the basis of specific activities. Statistical analysis was performed using Student's $t$ test. All values are expressed as mean \pm 1 SD.

In order to determine the effects of duration of incubation, dilution of labeled substrate, and size of endogenous fatty acid pool size, a series of preliminary studies were conducted as follows. Lung slices were incubated in flasks which contained the usual buffered medium and ${ }^{14} \mathrm{C}$-palmitate prepared as described. These flasks were incubated for 30-, 60-, and 90-min intervals after which phosphatidylcholine was recovered and analyzed for isotope uptake. The results for rate of uptake between the aminophylline and control lungs were compared and tested for linearity of palmitate incorporation by least squares regression analysis. In some instances, the slices were also pulse labeled with ${ }^{14} \mathrm{C}$-palmitate for $20 \mathrm{~min}$. The tissue was then rinsed free of labeled medium and reincubated in medium which contained an equal amount of nonradioactive palmitate serving as a cold chase. Incubation was then continued for 20 - and 40 -min intervals after which the tissue was prepared for lipid analysis as described later in this paper.

In another preliminary experiment, lung slices from the two study groups were incubated in a medium containing ${ }^{14} \mathrm{C}$-palmitate which in one case was prepared to a specific activity of 5.6 $\mu \mathrm{Ci} / \mu$ mole and in another at $0.56 \mu \mathrm{Ci} / \mu$ mole. The content of total palmitate was in turn adjusted to 1.0 or $10.0 \mu$ moles/flask, respectively, representing a 10 -fold dilution of labeled palmitate in the latter case.

\section{RESULTS}

A number of preliminary studies were conducted in order to establish suitable incubation conditions to be used in this study, i.e., a time period over which incorporation of precursors is linear and adequate amounts of precursor radioactivity are incorporated. The possibility exists in such studies that large changes in the availability or pool size of substrates could affect the apparent rates at which substrates are utilized. Specifically, the introduction of a labeled precursor to variable endogenous precursor pools could result in misleading interpretation of levels of isotope incorporation into lipids due to variable dilution. These conditions could result, for example, from the lipolytic action of aminophylline which may liberate large amounts of free fatty acids from triglycerides. Figure 1 shows the incorporation of palmitate into phosphatidylcholine over a $90-\mathrm{min}$ incubation interval. Incorporation is linear over $90 \mathrm{~min}$ and no difference in linearity exists between control and aminophylline treated lungs. In some instances, incubation was continued to $120 \mathrm{~min}$ (data not shown) with incorporation remaining linear. The results for the pulse labeling experiments are shown on the lower part of Figure 1. During the cold chase, the disappearance of labeled phosphatidylcholine was significantly slower in the aminophylline treated lungs. Saturated phosphatidylcholine was prepared from aliquots of total phosphatidylcholine and the results for palmitate incorporation and decay of labeled saturated phosphatidylcholine are shown in Figure 1. Uptake was again linear over $90 \mathrm{~min}$, with the rate of uptake being greater for the aminophylline treated group $(P<0.05)$. No significant differences in decay rates between the study groups were found although the level of labeled saturated phosphatidylcholine remained consistently higher in the experimental samples.

Table 1 presents the data obtained when lung slices were incubated with ${ }^{14} \mathrm{C}$-palmitate, specific activity of $5.6 \mu \mathrm{Ci} / \mu$ mole, diluted with cold palmitate to concentrations of either 1.0 or 10.0 $\mu$ moles/flask. In samples containing the larger amounts of substrate, incorporation into phosphatidylcholine increased approximately five-fold. This is likely due to greater substrate availability and is in agreement with the findings of Shaw and Rhoades (28). Furthermore, no differences were found between the two study groups indicating that large variations in endogenous fatty acids pools either do not occur, or do not necessarily affect uptake into phosphatidylcholine.

The 90-min interval was used for all subsequent experiments because substrate incorporation remains linear during this period for the major phospholipid fraction of lung, and standard deviations are considerably less than those observed after $120 \mathrm{~min}$ of incubation.

After the $90-\mathrm{min}$ incubation period, mean glucose consumption in the control lungs was $8.7 \pm 1.2$ while in aminophylline treated lungs it was $8.9 \pm 1.3 \mu$ moles/g tissue. Thus, there is no significant difference in the amounts of glucose utilized. Similarly, there was no significant difference between the amounts of glucose oxidized, $126.5 \pm 29.5$ and $126.2 \pm 38.2 \mu$ moles $/ g$ tissue for controls and aminophylline treated lungs, respectively. Lactate production was $11.7 \pm 3.5 \mu \mathrm{moles} / \mathrm{g}$ tissue in control and $12.0 \pm 4.0 \mu \mathrm{moles} / \mathrm{g}$ tissue in the aminophylline group. These values are not significantly different.

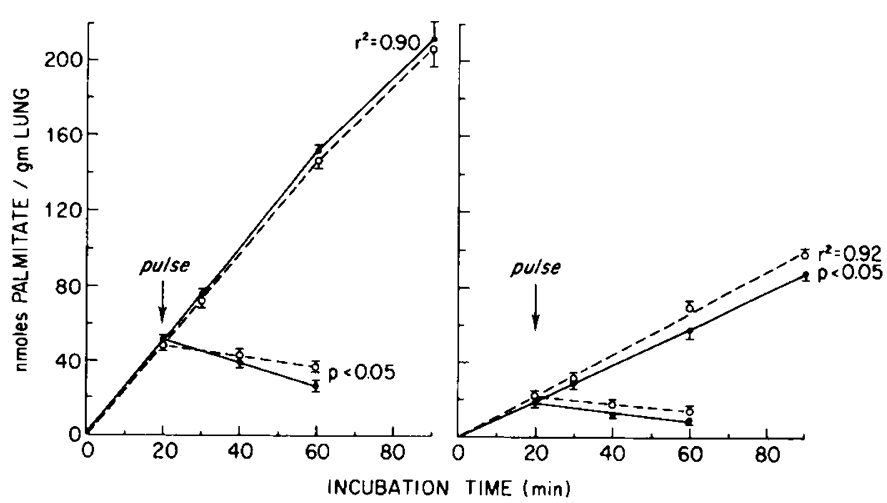

Fig. 1. A: Incorporation of palmitate into phosphatidylcholine by fetal lung slices over $90-\mathrm{min}$ incubation. Pretreatment with aminophylline denoted by broken line and controls denoted by solid line. Figure $1 \mathrm{~B}$ : Incorporation of palmitate into saturated phosphatidylcholine by fetal lung slices 90-min incubation. Pretreatment with aminophylline denoted by broken line and controls denoted by solid line. (See text for details). Two litters from each treatment group were studied in separate experiments, and the values represent means and SD from triplicate measurements at each time interval in a given study group.

Table 1. Incorporation of palmitate into phosphatidylcholine in fetal lung slices expressed as nmoles incorporated per $g$ tissue over 60 min incubation

\begin{tabular}{lcc}
\hline & $\mathrm{A}^{1}$ & $\mathrm{~B}^{2}$ \\
\hline Control & $124.8 \pm 24.4$ & $545 \pm 55.2$ \\
Aminophylline & $119.6 \pm 18.6$ & $569 \pm 60.3$ \\
\hline
\end{tabular}

'A-1.0 mole palmitate per flask at $5.6 \mu \mathrm{Ci} / \mu$ mole.

${ }^{2} \mathrm{~B}-10.0$ mole palmitate per flask at $0.56 \mu \mathrm{Ci} / \mu$ mole. 
Table 2 lists the amounts of lipids recovered from both control and aminophylline treated lungs. Significant increases were found in the content of phosphatidylcholine, saturated phosphatidylcholine, phosphatidylglycerol, phosphatidylinositol, and free fatty acids in the aminophylline-treated group. A reduction was found for triglycerides. Notwithstanding the increases found for nearly all the individual phospholipids, no significant difference was measured for total phospholipid content.

Table 3 presents the data for palmitate uptake into lipids of fetal lung slices. Uptake is significantly increased in saturated phosphatidylcholine and phosphatidylinositol fractions. A significant reduction is found for triglycerides and for the neutral lipids. The results for oleate uptake are presented in Table 4. It appears that although uptake into phosphatidylcholine is unchanged, there is a significant increase for phosphatidylglycerol and phosphatidylinositol in the aminophylline-treated group. Again, there was reduced uptake into the neutral lipids. Comparing the ratio of palmitate to oleate incorporated into the respective lipids reveals that over $50 \%$ more palmitate than oleate is incorporated into phosphatidylcholine while $30 \%$ more oleate than palmitate is incorporated into phosphatidylglycerol.

The results for glucose uptake into lung lipids are shown in
Table 5. Aminophylline treatment results in increased conversion of glucose to saturated phosphatidylcholine, but not total phosphatidylcholine. Increases are also found for sphingomyelin and phosphatidylinositol. The extent of glucose conversion into the fatty acid and glycerol moieties of phosphatidylcholine and neutral lipids was examined after methanolysis of the respective lipid fractions. The ratio of fatty acids to glycerol for control lung phosphatidylcholine was $1.45 \pm 0.29$ and for aminophylline, 0.85 $\pm 0.19(P<0.01)$. Because the total amount of phosphatidylcholine produced during this interval was unchanged, most of the labeled glucose was probably directed toward formation of the glycerol backbone of phosphatidylcholine while fatty acids were more likely derived from endogneous pools. The fatty acid to glycerol ratio for the neutral lipids was $0.72 \pm 0.11$ and $0.65 \pm$ 0.11 for the control and aminophylline groups, respectively. These values are not significantly different and may reflect higher levels of reesterification in addition to increased lipolysis in the aminophylline-treated group. Increased lipolysis and reesterification have been reported to occur after increases in cAMP concentrations in hepatocytes (17). The free fatty acids have also been shown to be potent feedback regulators of triglyceride lipase and adenylate cyclase (9).

Table 2. Lipids recovered from 27-day fetal lung slices

\begin{tabular}{lcccc}
\hline & $\mathrm{N}$ & Control & Aminophylline \\
\hline Phospholipids & $20^{1}$ & $9.64 \pm 0.67$ & $10.24 \pm 0.81$ & $\mathrm{NS}$ \\
Phosphatidylcholine & $20^{1}$ & $4.59 \pm 0.54$ & $5.16 \pm 0.64$ & $P<0.05$ \\
Saturated phosphatidylcholine & $20^{1}$ & $2.20 \pm 0.08$ & $2.30 \pm 0.08$ & $P<0.05$ \\
Phosphatidylglycerol & $20^{1}$ & $2.27 \pm 0.18$ & $2.91 \pm 0.10$ & $P<0.01$ \\
Phosphatidylinositol & $20^{1}$ & $0.24 \pm 0.03$ & $0.27 \pm 0.02$ & $P<0.02$ \\
Sphingomyelin & $20^{1}$ & $0.89 \pm 0.18$ & $1.02 \pm 0.09$ & $\mathrm{NS}$ \\
Neutral lipids & $20^{2}$ & $2.02 \pm 0.19$ & $2.14 \pm 0.22$ & $\mathrm{NS}$ \\
Triglycerides & $20^{2}$ & $0.67 \pm 0.07$ & $0.48 \pm 0.07$ & $P<0.01$ \\
Free fatty acids & $20^{2}$ & $0.16 \pm 0.04$ & $0.19 \pm 0.01$ & $P<0.05$ \\
\hline
\end{tabular}

${ }^{1}$ Expressed as $\mu \mathrm{mole} / \mathrm{g}$ lung (see text for details).

${ }^{2}$ Expressed as $\mathrm{mg} / \mathrm{g}$ lung (see text for details).

Table 3. Uptake of palmitate into the lipids of fetal lung slices (expressed as nmoles incorporated/g tissue/90 min)

\begin{tabular}{lcccc}
\hline & N & Control & Aminophylline & \\
\hline Neutral lipids & 20 & $73.7 \pm 10.4$ & $52.3 \pm 3.9$ & $P<0.001$ \\
Triglycerides & 20 & $16.8 \pm 0.9$ & $13.2 \pm 1.1$ & NS \\
Phosphatidylcholine & 20 & $204.0 \pm 17.3$ & $201.0 \pm 19.0$ & N.0 \\
Saturated Phosphatidylcholine & 20 & $89.0 \pm 6.0$ & $95.0 \pm 7.1$ & NS \\
Sphingomyelin & 20 & $9.4 \pm 2.4$ & $12.1 \pm 0.7$ & NS \\
Phosphatidylglycerol & 20 & $10.8 \pm 2.4$ & $2.9 \pm 0.1$ & $P<0.05$ \\
Phosphatidylinositol & 20 & $2.3 \pm 0.5$ & & \\
\hline
\end{tabular}

Table 4. Uptake of oleate into the lipids of fetal lung slices (expressed as nmoles $/ \mathrm{g}$ tissue/90 min)

\begin{tabular}{llccc} 
& $\mathrm{N}$ & Control & Aminophylline & \\
\hline Neutral lipids & 20 & $74.0 \pm 7.0$ & $64.0 \pm 9.1$ & $P<0.02$ \\
Phosphatidylcholine & 20 & $132.5 \pm 16.2$ & $126.4 \pm 11.9$ & NS \\
Sphingomyelin & 20 & $3.3 \pm 0.9$ & $3.1 \pm 0.7$ & NS \\
Phosphatidylglycerol & 20 & $13.9 \pm 1.1$ & $17.9 \pm 4.7$ & $P<0.02$ \\
Phosphatidylinositol & 20 & $9.8 \pm 0.4$ & $12.6 \pm 0.6$ & $P<0.001$ \\
\hline
\end{tabular}

Table 5. Uptake of glucose into the lipids of fetal lung slices (expressed as nmoles $/ \mathrm{g}$ tissue $/ 90$ min)

\begin{tabular}{lllcc}
\hline & N & Control & Aminophylline & \\
\hline Neutral lipids & 16 & $21.6 \pm 1.6$ & $20.4 \pm 2.5$ & NS \\
Phosphatidylcholine & 20 & $32.4 \pm 3.7$ & $33.7 \pm 3.9$ & NS \\
Saturated phosphatidylcholine & 20 & $12.6 \pm 0.9$ & $14.6 \pm 1.6$ & $P<0.05$ \\
Sphingomyelin & 18 & $24.2 \pm 2.2$ & $30.4 \pm 2.3$ & P \\
Phosphatidylglycerol & 18 & $28.4 \pm 3.5$ & $31.8 \pm 3.7$ & NS \\
Phosphatidylinositol & 18 & $22.8 \pm 1.4$ & $33.4 \pm 4.2$ & $P<0.001$ \\
\hline
\end{tabular}


Table 6. Glycogen content and specific activity in fetal lung slices (expressed as $\mathrm{mg}$ equivalent glucose $/ \mathrm{g}$ tissue) ${ }^{1}$

\begin{tabular}{lccc}
\hline & Before incubation & $\begin{array}{c}\text { After } 90 \mathrm{~min} \\
\text { incubation }\end{array}$ & $\begin{array}{c}\text { Specific } \\
\text { Activity }^{2}\end{array}$ \\
\hline Control & $5.23 \pm 0.75^{3}$ & $3.97 \pm 0.143$ & $48.8 \pm 5.0^{4}$ \\
Aminophylline & $4.40 \pm 0.69^{3}$ & $2.60 \pm 0.93^{3}$ & $38.8 \pm 8.7^{4}$
\end{tabular}

' All comparisons are between control and aminophylline.

${ }^{2}$ Specific activity expressed as nmoles glucose incorporated per $\mathrm{mg}$ glycogen, $n=18$.

${ }^{3} P<0.01$

${ }^{4} P<0.05$

The content of glycogen recovered from lungs both before and after incubation, and amounts of ${ }^{14} \mathrm{C}$-glucose incorporated into lung glycogen are shown in Table 6. Aminophylline treatment results in a lowered tissue glycogen content, and this reduction continues during the 90 -min incubation period. Glycogen synthesis is decreased, demonstrated by the lower specific activity of glycogen recovered from aminophylline treated lung slices.

\section{DISCUSSION}

Aminophylline may exert its effects through a combination of mechanisms which are also enhanced by cortisol. There may be directed utilization of glucose and/or glycogen as well as fatty acids in the fetal lung which results in increased phospholipid production. This is manifested primarily by increased saturated phosphatidylcholine formation as well as at least two minor acidic phospholipids.

The data presented indicate that large changes in the pool of fatty acids do not account for the amounts of phosphatidylcholine synthesized nor for the effects of it in our lung slice model. Evidence has been provided that aminophylline does not alter the pool of palmitate in lungs to the extent that incorporation of this precursor into phosphatidylcholine is significantly changed. A 10fold change in size of the palmitate pool is metabolized similarly in control and aminophylline treated lung slices. Aminophylline which is known to produce lipolysis in other tissues would be expected to increase the size of the endogenous pool of fatty acids and would, therefore, be more likely to diminish the apparent rate of incorporation of palmitate into saturated phosphatidylcholine. The utilization of palmitate in the lung is the result of a number of enzymatic processes involved in both de novo synthesis and remodeling of phospholipids. De novo synthesis of phosphatidylcholine is thought to utilize primarily mono- and dienoic diacylglycerols as the substrates for cholinephosphotransferase during the final enzymatic step in biosynthesis (25). On this basis, one would expect approximately equimolar incorporation of saturated and unsaturated fatty acids into phosphatidylcholine. From the data presented in Tables 3 and 4, a ratio of palmitate to oleate of approximately 1.5 is derived. This indicates an enrichment of saturated fatty acids in phosphatidylcholine which would result from transacylation reactions in the lung to produce disaturated phosphatidylcholine $(1,15)$. The increases in saturated phosphatidylcholine found in the aminophylline treated fetal lungs suggest either an augmented remodeling of phosphatidylcholine, or a slower turnover of newly synthesized saturated phosphatidylcholine, possibly by sequestration into lamellar bodies. The data for decay rates in Figure I suggest the latter possibility, but further studies to measure effects on the phosphatidylcholine remodeling pathways are necessary.

The lung slice model offers numerous advantages compared with cell culture and homogenization techniques because it allows similar intercell reactions as would be found in intact tissues. It provides, in addition, a system in which pool sizes of substrates can be measured and manipulated. O'Neil and Tierney (23) have demonstrated no significant differences in glucose metabolism between lung slices and isolated perfused lung, indicating that the lung slice model may be particularly useful in the study of phospholipid metabolism when glucose is used as a substrate.
These advantages outweigh the lack of vascular supply of substrates present in vivo and in isolated perfused lungs but absent in cell culture. Effects of loss of alveolar lining material into the incubation medium are currently being studied.

Recently, Hallman and Gluck (14) demonstrated that measurement of phosphatidylglycerol and phosphatidylinositol is a good indicator of fetal lung maturity especially when analyzing amniotic fluid or tracheal washes. In the rabbit, phosphatidylinositol reaches maximum concentrations at term and then declines after birth while phosphatidylglycerol reaches maximum concentrations after birth. In the human, the reverse is the case and, therefore, the presence of phosphatidylglycerol has been proposed as an indicator for maturity in the human (13). These data show that the fetal rabbit lung is actively producing phosphatidylinositol by the 27th day of gestation, and is even more active after aminophylline administration. Unlike Hallman and Gluck (14), the authors measured the content and production of phosphatidylglycerol and phosphatidylinositol in fetal lung tissue of the rabbit and not in tracheal washings or lamellar bodies. It is possible that later in development or after birth, the content of these phospholipids increase in lamellar bodies which may later be released into the airspaces. Oleate is incorporated into both these phospholipids more readily than is palmitate and, consequently, oleate incorporation may serve as a more sensitive indicator of the production of these phospholipids. Analysis of the fatty acid composition of these acidic phospholipids indicates that mono- and dienoic fatty acids are the major components.

A possible route for the biosynthesis of phosphatidylcholine from glucose, and enzymatic steps which may be influenced by cortisol (or aminophylline) have been previously described (12).

Increases in intracellular cAMP concentrations result from both aminophylline and cortisol administration. This is brought about at least in part by inhibition of cAMP phosphodiesterase activity. Both cortisol and aminophylline have been shown to inhibit cAMP phosphodiesterase at low concentrations $(5,21,26)$. Increases in cAMP concentrations have been reported to influence numerous enzymes involved in both carbohydrate and lipid metabolism (24). Findings such as these implicate cAMP as an underlying agent involved in the regulation of catabolic and synthetic pathways leading to surfactant phospholipid production.

These results contribute further evidence that aminophylline is an effective agent for accelerating maturation of fetal lungs. Thus, further studies of the potential usefulness of antepartum administration of aminophylline to enhance survival of infants with immature lung function are warranted.

\section{REFERENCES AND NOTES}

I. Abe, M., and Ohno. K.: The formation of lecithin from lysolecithin in rat lung supernatant. Biochim. Biophys. Acta, 280: 275 (1972).

2. Barrett, C. T., Sevanian. A.. Phelps, D. L.. Gilden. C., and Kaplan, S. A.: Effects of cortisol and aminophylline upon survival, pulmonary mechanics, and secreted phosphatidylcholine of prematurely delivered rabbits. Pediatr. Res., 12 38 (1978).

3. Barrett. C. T., Sevanian. A., Lavin. N., and Kaplan, S. A.: Role of adenosine 3' 5 -monophosphate in maturation of fetal lungs. Pediatr. Res., 10:621 (1976).

4. Bartlett. G. R.: Phosphorus assay in column chromatography. J. Biol. Chem. 234: 466 (1959)

5. Beavo. J. A., Rogers, N. L., Crofford, O. B.. Baird. C. E., Hardman, J. G. Sutherland. E. W., and Newman. E. V.: Effects of phosphodiesterase inhibitors on cyclicAMP levels and on lipolysis. Ann. NY Acad. Sci., 185: 129 (1971)

6. Bligh, E. G., and Dyer. W. J.: A rapid method of total lipid extraction and purification. Can. J. Biochem. Physiol., 37: 911 (1959)

7. Carson, S. H., Taeusch, H. W., and Avery, M. E.: Inhibition of lung cell division rate after hydrocortisone injection into fetal rabbits. J. Appl. Physiol., 34: 660 (1973).

8. Davidson. M. B., and Berliner, J. A.: Acute effects of insulin on carbohydrate metabolism in rat liver slices: independence from glucagon. Am. J. Physiol. 227: 79 (1974)

9. Fain, J. N., and Shepherd, R. E.: Free fatty acids as feedback regulators of adenylate cyclase and cyclic $3^{\prime}: 5^{\prime}$-AMP accumulation in rat fat cells. J. Biol Chem.. 250: 6586 (1975)

10. Farrell, P. M., and Avery, M. E.: Hyaline membrane disease. Amer. Rev. Respir. Dis., 111: 657 (1975).

11. Farrell, P. M., and Zachman, R. D.: Induction of choline phosphotransferase and tecithin synthesis in the fetal lung by corticosteroids. Science, 179: 297 (1973). 
12. Gilden. C.. Sevanian, A.. Tierney, D. F.. Kaplan. S. A., and Barrett, C. T.: Regulation of fetal lung phosphatidylcholine synthesis by cortisol: Role of glycogen and glucose. Pediatr. Res., 11: 845 (1977).

13. Hallman. M. Feldman. B.. and Gluck. L.: RDS: the absence of phosphatidylglycerol in surfactant. Pediatr. Res., 9: 396 (1975).

14. Hallman, M., and Gluck. L.: Phosphatidylglycerol in lung surfactant. III. Possible modifier of surfactant function. J. Lipid. Res., 17: 257 (1976).

15. Hallman, M., and Raivio. K.: Studies on the biosynthesis of disaturated lecithin of the lung: the importance of the lysolecithin pathway. Pediatr. Res.. 8: 874 of the
(1974).

16. Hohorst, H. J.: L-(+)-lactate determination with lactic dehydrogenase and DNP. In: H. U. Bergmeyer: Methods of Enzymatic Analysis. pp. 226-270 (Academic Press. New York. 1965)

17. Homcy. C. J., and Margolis, S.: Fatty acid oxidation and esterification in isolated rat hepatocytes: regulation by dibutyryl adenosine $3^{\prime}, 5^{\prime}$-cyclic monophosphate. J. Lipid Res., 141:678 (1973).

18. Howard. E.: Reduction in size and total DNA of cerebrum and cerebellum in adult mice after corticosterone treatment in infancy. Exp. Neurol., 22: 191 (1968).

19. Karotkin. E. H., Kido, M., Cashore, W. J., Redding. R. A., Douglas. W. J., Stem and Oh, $W$. Acceleration of fetal lung maturation by aminophylline in pregnant rabbits. Pediatr. Res., 10: 722 (1976).

20. Kotas. R. V.., and Avery. M. E.: Accelerated appearance of pulmonary surfactant in the fetal rabbit. J. Appl. Physiol., 30: 358 (1971)

21. Lavin, N.. Rachelefsky, G.. and Kaplan. S. A.: Inhibition of cyclic-AMP phosphodiesterase in human lymphocytes by physiological concentrations of hydrocortisone. Hormone Metabol. Res., 7: 253 (1975).

22. Liggins, G. C.. and Howie, R. N.: A controlled trial of antepartum glucocorticoid treatment for prevention of respiratory distress syndrome in premature infants. Pediatrics, 50: 515 (1972).

23. O'Neil. J. J., and Tierney, D. F.: Rat lung metabolism: glucose utilization by isolated perfused lungs and tissue slices. Amer. J. Physiol., 226: 867 (1974).
24. Robison, G. A., Butcher, R. W., and Sutherland, E. W.: Cyclic AMP. pp. 17-29 (Academic Press. New York. 1971).

25. Sarzala, M. G.. and Van Golde. L. M. G.: Selective utilization of endogenous unsaturated phosphatidylcholines and diacylglyceroles by choline phosphotransferase of mouse lung microsomes. Biochim. Biophys. Acta. 44I: 423 (1976).

26. Schmidtke, J., Wienker. T., Flugel. M.. and Engel. W.: In vitro inhibition of cyclic AMP phosphodiesterase by cortisol. Nature. 262: 593 (1976).

27. Shapiro, S.: Some physiologic. biochemical, and behavioral consequences of neonatal hormone administration. Cortisol and Thyroxin. Gen. Comp. Endocrinol., 10: 214 (1968).

28. Shaw, M. E.. and Rhoades, R. A.: Substrate metabolism in the perfused lung: response to changes in circulating glucose and palmitate levels. Lipids, 12: 930 (1977).

29. Shimojo, T. M. Abe, M., and Ohta, M.: A method for determination of saturated phosphatidylcholine. J. Lipid Res., 15: 545 (1974).

30. Taeusch. H. W.. Frigoletto. F., Kitzmiller. J.. Avery, M. E.. Hehre. A.. Fromm. B. Lawson. E., and Neff. A. K.: Risk of respiratory distress syndrome and maternal infection after prenatal dexamethasone treatment. Pediatr. Res.. 12: 535 (1978)

31. Van Handel. E.: Estimation of glycogen in small amounts of tissue. Anal. Chem., 11: 256 (1965).

32. Washko, M. E., and Rice. E. W.: Determination of glucose by an improved enzymatic procedure. Clin. Chem., 7: 542 (1961).

33. This research was supported in part, by grants from the California Research and Medical Education Fund of the California Lung Association. a General Research Support Grant from the University of California at Los Angeles, the Kroc Foundation and United States Public Health Service HD-12197.

34. Requests for reprints should be addressed to: Alex Sevanian. Ph.D.. Department of Pediatrics, UCLA School of Medicine. Los Angeles. CA 90024 (USA).

35. Received for publication August 8, 1978.

36. Accepted for publication November 27, 1978. 